\title{
On Curved Diffraction-gratings
}

This content has been downloaded from IOPscience. Please scroll down to see the full text. 1882 Proc. Phys. Soc. London 5243

(http://iopscience.iop.org/1478-7814/5/1/329)

View the table of contents for this issue, or go to the journal homepage for more

Download details:

IP Address: 165.123.34.86

This content was downloaded on 07/09/2015 at 19:51

Please note that terms and conditions apply. 
XXVIII. On Curved Diffraction-gratings, By R. T. GLAZEвRоок, M.A., F.R.S., Fellow and Lecturer of T'rinity College, Demonstrator at the Cavendish Laboratory, Cambridge*.

P'ROF. Rowland has described the appearances presented when a beam of light, after passing through a slit, falls on " grating ruled on a cylindrical surface, and has given a very elegant construction for determining the position of the diffracted foci in the case in which the principal section of the grating is a circle and the source of light is placed at its centre of curvature. The mathematics of the subject have been dealt with still more recently by M. Mascart (Journal de ['hysique, January 1883) and Mr. W. Baily (Phil. Mag. M:irch 1883). The object of the present paper is to carry the discussion somewhat further.

Prof. Rowland claims for his gratings that they enable him to form a pure spectrum without the use of lenses, and hence hive an immense advantage over those hitherto employed. It must, however, be remembered that the formulæ obtained to give the position of the diffracted spectra are only true to a first approximation, that the spectra formed and the source of light are to one another in the relation of the conjugate geometrical foci of a lens or mirror. All the waves which arrive at any one point of the spectrum are not in exactly the sams phase. Aberration effects are produced, and have to be considered just as in the ordinary theory of lenses or mirrors. Now, if a plane wave of light fall on a plane grating, and the effects be observed on a screen at an infinite distance behind the grating, the spectrum formed is perfectly pure; all the red light, after passing the grating, is definitely brought to a focus at one point; there is no alerration, so far at least as the grating is concerned. Of course the difficulty is to obtain the plane wave and the screen at an infinite distance. If the source of light be placed at the principal focus of a collimating lens, the emergent wave differs from a plane by quantities depending on the aberration of the lens; while if the diffracted beam is received on a second lens and a screen he placed in the focal plane of that lens, the screen would

* Read April 14, 1883. 
practically be at an infinite distance from the grating but for the aberration produced by the lens.

So far, then, as definition merely is concerned, we have to compare the aberration effects produced by these lenses with those caused by the curvature of the grating. Of course a reflexion grating used without lenses has an immense advanfage for cxperiments on the violet or ultra-violet rays which are absorbed by glass.

In considering the aberration, then, we shall follow the mothod adopted hy Lord Rayleigh in his paper on "Investigations in Optics, with special reference to the Spectroscope. Aherration of Lenses and Prisms" (Phil. Mag. January 1880). Let $Q \mathrm{~A}, \mathrm{Q} \mathrm{P}$ be two aljacent rays diverging from a point $Q$ and falling on the concave side. of a circlo A P, centre ). Let $\mathrm{QAO}=\phi$, $\mathrm{A} \cap \mathrm{P}=\omega, \quad \mathrm{QA}=u$, $\mathrm{OA}=a$. Then

$$
\begin{aligned}
& \mathrm{QAP}=\phi+\frac{\pi}{2}-\frac{\omega}{2}, \\
& \mathrm{AP}=2 a \sin \frac{\omega}{2} .
\end{aligned}
$$

Figr. ].

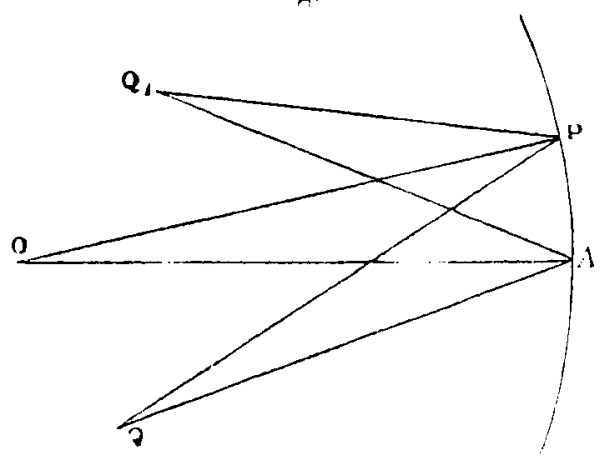

Hence

$$
\mathrm{QP}^{2}=u^{2}+4 a^{2} \sin ^{2}{ }_{2}^{\omega}-4 a u \sin \frac{\omega}{2} \sin \left(\frac{\omega}{2}-\phi\right) ; .
$$

and, expanding as far as $\omega^{3}$, wo find

$$
\begin{aligned}
Q \mathrm{P}= & u+a \omega \sin \phi-\frac{a \omega^{2}}{2}\left(\cos \phi-{ }_{u}^{a} \cos ^{2} \phi\right) \\
& -\frac{a \omega^{3} \sin \phi}{2}\left(\frac{1}{3}-\frac{a}{u} \cos \phi+\frac{a^{2}}{u^{2}} \cos ^{2} \phi\right)+\ldots
\end{aligned}
$$

Again, lat $Q_{1}$ be another point on the other side of the normal $\mathrm{OA}$, and let $\mathrm{Q}_{1} \mathrm{~A}=u^{\prime}, \mathrm{Q}_{1} \mathrm{AO}=\psi$. Then

$$
\begin{aligned}
Q_{1} P= & u^{\prime}-a \omega \sin \psi-\frac{a \omega^{2}}{2}\left(\cos \psi-\frac{a}{u^{\prime}} \cos ^{2} \psi\right) \\
& +\frac{a \omega^{3} \sin \psi}{2} \psi\left(\frac{1}{3}-\frac{a}{u^{\prime}} \cos \psi+\frac{a^{2}}{\left.u^{\prime 2} \cos ^{2} \psi\right)}\right.
\end{aligned}
$$


Suppose now that A is a point on one line of the grating; and $\mathrm{P}$ a corresponding point on some other line. Then waves from $Q$ diffracted at $A$ and $P$ respectively will reach $Q_{1}$ in the same phase if $Q P+Q_{1} P=Q A+Q_{1} A \pm n \lambda, \lambda$ being the wave. length. That is, if

$$
\begin{aligned}
& a \omega(\sin \phi-\sin \psi) \\
& \quad-\frac{a \omega^{2}}{2}\left\{\cos \phi+\cos \psi-a\left(\frac{\cos ^{2} \phi}{u}+\frac{\cos ^{2} \psi}{u^{\prime}}\right)\right\} \\
& \quad-\frac{a \omega^{3}}{2}\left\{\sin \phi\left(\frac{1}{3}-\frac{a}{u} \cos \phi+\frac{a^{2}}{u^{2}} \cos ^{2} \phi\right)\right. \\
& \left.\quad-\sin \psi\left(\frac{2}{3}-\frac{a}{u^{\prime}} \cos \psi+\frac{a^{2}}{u^{\prime 2}} \cos ^{2} \psi\right)\right\}= \pm n \lambda .
\end{aligned}
$$

This is equivalent to Mr. Baily's formula carried to the next degree of approximation; and his results are obtained by neglecting the term in $\omega^{3}$ and taking $\phi$ and $\psi$ to satisfy the equation

$$
\sin \phi-\sin \psi= \pm \frac{n \lambda}{a \omega}, \quad . \quad . \quad \text {. . }
$$

and then $u$ and $u^{\prime}$ to satisfy

$$
\cos \phi+\cos \psi-a\left(\frac{\cos ^{2} \phi}{u}+\frac{\cos ^{2} \psi}{u^{\prime}}\right)=0 . \quad .
$$

To consider the aberration we have two cases before us. Let us suppose (1) that equation (5) holds, and determine the value $u_{1}^{\prime}$, say of $u^{\prime}$, considering the terms in $\omega^{3}$ in equation (4). This will give us what we may call the longitudinal aberration.

In the second case we shall suppose equation (6) to hold, and determine the value for $\psi$ which satisties (4) to the same approximation. 'This will give us the lateral aberration.

In the general case equation (4), as it stands, really determines the locus of the inage of $Q$ formed by diffraction at the two lines $A$ and $P$; and this locus is clearly an byperbola, with $\mathrm{A}$ and $\mathrm{P}$ as foci. Waves diffracted at $\mathrm{A}$ and $\mathrm{P}$ respectively will arrive in the same phase at any point of this hyperbola. For every point such as $\mathrm{P}$ on the grating an byperhola possessing similar properties can be drawn. If all these hyperbolas meet in a point, then that point is really a focus for waves diverging from $Q$; they all are in the same phase when they meet there. This is the case if the grating be plane and $Q$ and $Q_{1}$ infinitely distant. If, however, the hyperbolas do not all meet in a point, there is really no focus in its strict sense, only a geometrical focus. If we neglect $\omega^{3}$ and higher 
terms, then the point given by (5) and (6) is to this approximation common to all the hyperbolas: it is the geometrical focus.

To discuss, then, the aberration in this case. Let $u_{1}^{\prime}=u^{\prime}+\delta u^{\prime}$, where $u^{\prime}$ satisfies (6), and suppose we neglect $\left.\overline{\delta u^{\prime}}\right|^{2}, a \omega \delta u^{\prime}$, and such terms. Then

$$
\begin{aligned}
\cos \phi+ & \cos \psi-a\left\{\frac{\cos ^{2} \phi}{u}+\frac{\cos ^{2} \psi}{u^{\prime}}\left(1-\frac{\delta u^{\prime}}{u^{\prime}}\right)\right\} \\
& +a \omega\left\{ \pm \frac{n \lambda}{3 a \omega}-\frac{a \sin \phi \cos \phi}{u}\left(1-\frac{a}{u} \cos \phi\right)\right. \\
& \left.+\frac{a \sin \psi \cos \psi}{u^{\prime}}\left(1-\frac{a}{u^{\prime}} \cos \psi\right)\right\}=0 .
\end{aligned}
$$

Thus

$$
\begin{aligned}
\dot{\delta} u^{\prime}=- & \frac{u^{\prime 2} \omega}{a \cos ^{2} \Psi}\left\{ \pm \frac{n \lambda}{3 a \omega}-\frac{a}{u} \sin \phi \cos \phi\left(1-\frac{a}{u} \cos \phi\right)\right. \\
& \left.+\frac{a}{u^{\prime}} \sin \psi \cos \psi\left(1-\frac{a}{u^{\prime}} \cos \phi\right)\right\} . \quad . \quad . \quad(7)
\end{aligned}
$$

Equation (7) determines the aberration in the general case. To determine the eftect of this in practice, let us suppose that we are considering the spectrum of the first order, so that the retardation of the light coming from two consecutive lines is just one wave-length ; and hence, if $\mathrm{P}$ be on the $k$ th line from $\mathrm{A}$, and $\sigma$ the distance between two lines, then the arc $\mathrm{AP}=$ $k \sigma=a \omega$, and $n=k$.

Let us suppose, further, that the origin of light is at the centre of curvature of the grating, so that $u=a, \phi=0$, and hence, taking the - ve sign in (6),

$$
\sin \psi=\frac{\lambda}{\sigma}, \cos \psi=\sqrt{\left(1-\frac{\lambda^{2}}{\sigma^{2}}\right)}, u^{\prime}=a \cos \psi=a \sqrt{\left(1-\frac{\lambda^{2}}{\sigma^{2}}\right)}
$$

Thus

$$
\delta u^{\prime}=+a \omega \frac{k \lambda}{3 a \omega}=+\frac{1}{3} k \lambda .
$$

Let $Q_{1}$ (fig. 2) be the point on the line given by $\sin \psi=\frac{\lambda}{\sigma}$, which is deternined by $u^{\prime}=a \cos \psi, Q^{\prime}$ being the point on that line at which light arrives in exactly the same phase from $A$ and $P$. Then $Q_{1} Q^{\prime}=\frac{1}{3} k \lambda$. And the angle $P Q_{1} A$ differs by only a small quantity from $\omega$; while, since $Q^{\prime} Q_{1}$ is small compared with $\mathrm{PQ}^{\prime}$, the angle $\mathrm{Q}_{1} \mathrm{PQ}^{\prime}$ is small compared with $\omega$. 
Hence, if $Q^{\prime} R$ be drawn at right angles to $P Q_{1}$, the light from $P$ arrives at $R$ in the same phase as at $Q^{\prime}$, and the difference

Fig. 2.

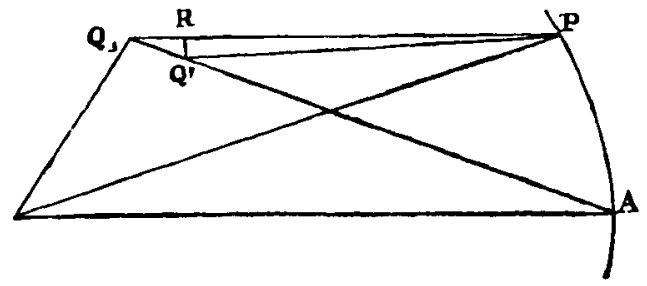

in phase at $Q_{1}$ between the waves coming from $A$ and $P$ is $\mathrm{Q}_{1} \mathrm{Q}^{\prime}-\mathrm{Q}_{1} \mathrm{R}=\mathrm{Q}_{1} \mathrm{Q}^{\prime}(1-\cos \omega)$; and to the same approximation this is equal to $\frac{1}{6} k \lambda \omega^{2}$. So that if we consider as well the light coming from a point $k^{\prime}$ lines below $\mathrm{A}$, the extreme difference of phase in the various waves which reach the point $Q_{1}$ is $\frac{1}{6}\left(k+k^{\prime}\right) \lambda \omega^{2} ; k+k^{\prime}$ will be the total number of lines in the grating.

Thus in one of Prof. Rowland's gratings we have

$$
\begin{aligned}
a & =213 \text { centim., } \\
\omega & =\frac{37}{2130} \text { about, } \\
k+k^{\prime} & =14250 ;
\end{aligned}
$$

and hence the difference in phase is about $7 \lambda / 10$. Hence the aperture of the grating is too large to give the best definition: for that purpose the difference of phase in the various secondary waves arriving at the point in question should not be greater than $\lambda / 4$.

We may conveniently express this difference of phase in terms of the number of lines, the radius of the grating, and the distance between the lines. Let $\sigma$ be the distance betw'een the lines; then

$$
\omega=\frac{\left(k+k^{\prime}\right)}{2 a} \sigma,
$$

and the difference of phase is

$$
\frac{1}{24} \times\left(k+k^{5}\right)^{3} \frac{\sigma^{2}}{a^{2}} \lambda .
$$

For good definition this difference of phase must not be greater than $\lambda / 4$. Since in the case above the difference of phase is $7 \lambda / 10$, we must reduce the number of lines, keeping the distance between them the same, in the ratio of $\sqrt[3]{10}$ to $\sqrt[3]{28}$, or 
rather more than 2 to 3 . Hence by covering up rather less than one third of the grating we should expect to produce better definition.

In another grating of Rowland's, $\sigma=\frac{1}{11400}$ centim., $a=520$ centim., $k+k^{\prime}=160,000$; and in this case the difference of phase comes out to be about $4.8 \times \lambda$. The grating is much too wide; it will require reducing in the ratio of $1: \sqrt[3]{19 \cdot 2}$, or about $3: 8$.

'To consider now the lateral aherration, using the same notation, describe a circle (fig. 3) through $Q_{1}$ with $A$ as centre. Light from $A$ arrives in the same phase at all points on this circle. Let $Q^{\prime}$ be the point on the circle at which the light arriving from $P$ is in the same phase as that from $A$, and let $\psi+\delta \psi$ be the angle $O A Q^{\prime}$,

Fig. 3.

and let $\psi, \phi, u$, and $u^{\prime}$ satisfy (5) and (6). Our fundamental equation (4) becomes, neglecting terms like $\omega^{2} \delta \psi$,

$$
\begin{aligned}
a \omega[\sin \phi & -\sin \psi-\delta \psi \cos \psi \\
& -\frac{\omega}{2}\left\{\cos \phi+\cos \psi-a\left(\frac{\cos ^{2} \phi}{u}+\frac{\cos ^{2} \psi}{u^{\prime}}\right)\right. \\
& \left.-\sin \psi \delta \psi\left(1-\frac{2 a \cos \psi}{u^{\prime}}\right)\right\} \\
& -\frac{\omega^{2}}{2}\left\{\sin \phi\left(\frac{1}{3}-\frac{a}{u} \cos \phi+\frac{a^{2}}{u^{2}} \cos ^{2} \phi\right)\right. \\
& \left.\left.-\sin \psi\left(\frac{1}{3}-\frac{a}{u^{\prime}} \cos \psi+\frac{a^{2}}{u^{\prime 2}} \cos ^{2} \psi\right)\right\}\right]= \pm n \lambda .
\end{aligned}
$$

Hence

$$
\begin{aligned}
\delta \psi\left\{\cos \psi-\frac{\omega}{2} \sin \psi\left(1-\frac{2 a \cos \psi}{u^{\prime}}\right)\right\} \\
+\frac{\omega^{2}}{2}\left[\sin \phi\left\{\frac{1}{3}-\frac{a}{u} \cos \phi\left(1-\frac{a}{u} \cos \phi\right)\right\}\right. \\
\left.\left.-\sin \psi\left\{\frac{1}{3}-\frac{a}{u^{\prime}} \cos \psi\left(1-\frac{a}{u^{\prime}} \cos \psi\right)\right\}\right]=0\right)
\end{aligned}
$$


and, to the approximation adopted in considering the longitudinal effect,

$$
\begin{array}{r}
\delta \psi=-\frac{\omega^{2}}{2} \sec \psi\left\{ \pm \frac{1}{3} \frac{n \lambda}{a \omega}-\frac{a}{u} \sin \phi \cos \phi\left(1-\frac{a \cos \phi}{u}\right)\right. \\
\left.+\frac{a}{u^{\prime}} \sin \psi \cos \psi\left(1-\frac{a}{u^{\prime}} \cos \psi\right)\right\} . \quad . \quad . \quad
\end{array}
$$

If, as before, $Q$ coincide with 0 , then $u=0, \phi=0, u^{\prime}=a \cos \psi$; and taking the negative sign, so that $\sin \psi=\frac{n \lambda}{a \omega}$,

$$
\begin{gathered}
\delta \psi=\frac{\omega \sec \psi \times n \lambda}{6 a}, \\
Q_{1} Q^{\prime}=u^{\prime} \delta \psi=\frac{n \lambda \omega}{6} .
\end{gathered}
$$

and

Thus, carrying our approximation as far as terms in $\omega^{3}$ in equation (4), we find that the position of the image formed, considering only two of the lines as producing diffraction effects, is not at $Q_{1}$ but $Q^{\prime}$, where $Q_{1} Q^{\prime}=\frac{n \lambda \omega}{6}$. Hence, if wo consider the whole grating, using the same notation as before, the breadth in a direction norinal to $A Q_{1}$ of the image formed will be comparable with $\frac{\left(k+k^{\prime}\right) \lambda \omega}{6}, \omega$ being the whole semiaperture. Expressing this in terms of the radius of the grating $a$ and the distance between the lines $\sigma$, we find the value $\frac{1}{6}\left(k+k^{\prime}\right)^{2} \frac{\lambda \sigma}{a}$. Thus the breadth of the image will depend on the square of the number of lines. In the grating first considered this quantity, $\frac{1}{6}\left(k+k^{\prime}\right) \lambda \omega$, is about $\frac{1}{500}$ of a centimetre for yellow light, while the distance between the $\mathrm{D}$ lines is about ${ }_{30}$ centim., or ten times as much; while in the second grating this lateral aberration is $\frac{1}{50}$ centim., the distance between the $\mathrm{D}$ lines being about seven times as great. If the size of this last grating be reduced to $\frac{3}{8}$ of what it actually is, the extreme lateral aberration will be reduced to $\frac{9}{64}$ or about $\frac{1}{7}$ of its actual value, thus becoming about $\overline{3} \frac{1}{5} \overline{0}$ of a centimetre, and the extreme difference of phase in the light of a given wavelength $\lambda$ reaching any point of the diffracted spectrum will never exceed $\lambda / 4$, the dispersion will remain unaltered, the definition and the brightness of the spectrum will both be increased.

It is clear that in both cuses the outer portion of the grating vol. $v$. 
not merely impairs the definition, but actually renders it less bright than before. For consider two points $\mathrm{P}_{1}, \mathrm{P}_{2}$ (fig. 4) equidistant from A, such that the difference in phase in the waves coming from $Y_{1}$ and $P_{2}$ to $Q_{1}$ is $\frac{\lambda}{2}$ (since the difference of phase for the extreme rays is in both cases greater than $\frac{\lambda}{2}$, these points Fig. 4.

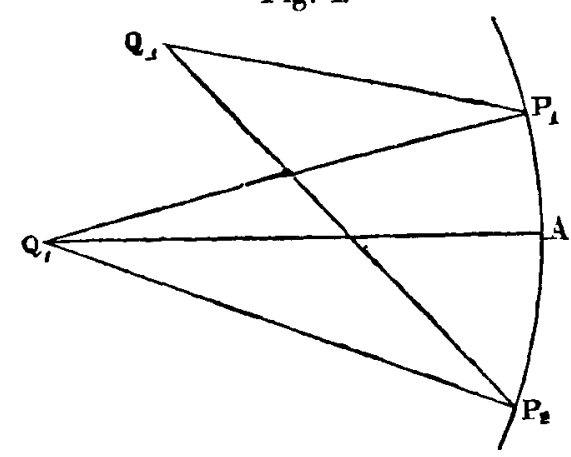
can be found). Then the light reaching $Q_{1}$ from above $P_{1}$ is opposite in phase to some of that which reaches $Q_{1}$ from between $\mathrm{P}_{2}$ and $A$, and tends to neutralize the effect of this; while similar results hold for light coming respectively from below $P_{2}$ and between $A$ and $P_{1}$. Thus a large aperture does not neccssarily mean that there is a large quantity of light at the focus. Exactly the same may happen in the case of a lens. Lord Rayleigh has shown that if $\alpha$ be the angular semi-aperture of the lens as viewed from the focus, and the curvatures of the lens be adjusted to reduce the longitudinal aberration to a minimum, $\alpha^{4}$ should not exceed $\lambda / f$. A similar course of reasoning shows us that if $\alpha^{4}$ is greater than $2 \lambda / f$, the light from the outer annulus of the lens will be opposite in phase to that from the central portions.

To compare, finally, the definition of the curved grating with that produced by a plane grating, and two lenses of equal focal length used as a collimator, and the object-glass of a telescope respectively, we can show (Parkinson, 'Optics,' $\S 130)$, that if $\alpha$ is the semi angular aperture of either of these lenses seen from its principal focus, $f$ its focal length, and the curvatures are adjusted to make the aberration of each lens a minimum, then the aberration is, for light of refractive index $1 \cdot 5, \frac{15}{7} \mathrm{fa}^{2}$; but, as quoted above, Lord Rayleigh has shown that the aberration shonld not. be greater than $\lambda / \alpha^{4}$. Hence $\alpha^{4}$ must not be greater than $\frac{7}{15} \frac{\lambda}{\dot{f}}$. 
In the case of the first of Prof. Rowland's gratings discassed above, the slit and eyepiece are at a distance of about 200 centim. from the grating. Let us suppose we are using two lenses of 200 centim. focal length, and inquire what their aperture may be to allow the condition above given to be satisfied. If $y$ be the radius of the lens, we have

$$
y^{4} \text { not }>\text { than } \frac{7 \times 8 \times 10^{6} \times 6}{15 \times 10^{5}} \text {. }
$$

Thus $y$ must not be greater than 3.8 centim. A lens of this aperture would just about admit the light from the whole of the actual grating 5 centin. $\times 7$ centim. in area if it were plane; whereas, without the lens, to obtain the best definition we are restricted to the use of about two thirds of the grating.

In the case of the other grating, we may, without increasing the size of the apparatus, use a lens of 500 centim. focal length; for good definition its aperture should not be more than

$$
\sqrt[4]{-\frac{7 \times 125 \times 10^{6} \times 6}{15 \times 10^{5}}}
$$

or about $7 \cdot 6$ centim. A lens of this aperture would enable us to use with the best advantage the whole of the grating if it were plane; whereas in the concave grating, for good definition we should only use about three eighths of the whole. It would seem, then, that in cases in which there is no objection to the use of glass (because of its absorbing qualities), a large grating may be used to greater advantage if it be ruled on a flat surface and properly chosen lenses be employed with it, than if the grating be curved.

It may be instructive to consider the subject briefly in another manner. Let $Q, Q_{1}$ (fig. 5) be any two points, and Fig. 5.

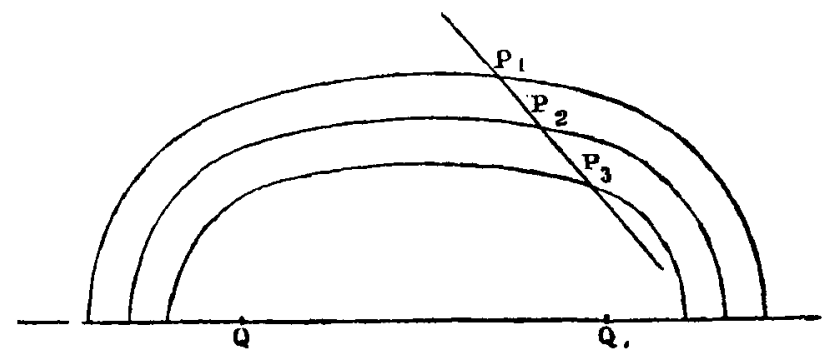

with $Q$ and $Q_{1}$ as foci describe a series of confocal ellipses; let the major axes of these ellipses increase in arithmetical progres- 
sion, and let the common difference be $\lambda$. Consider a spherical wave diverging from $Q$ and reflected at any point of any one of these ellipses; all the reflected light will reach $Q_{1}$ in the same phase. Take any surface $\mathrm{P}_{1}, \mathrm{P}_{2}$, \&c. cutting the ellipses in $\mathrm{P}_{1}, \mathrm{P}_{2}$ \&c., and suppose it capable of reflecting light at these points and incapable of so doing elsewhere. All the light from $Q$ which falls on this surface at these points will be reflected to $Q_{1}$, and the various waves will reach $Q_{1}$ in the same phase. If now $Q$ be the section of a slit normal to the paper, $P_{1} P_{2}$ \&c. that of a polished cylindrical surface whose generators are normal to the paper, and lines be ruled on this surface to block out the spaces $\mathrm{P}_{1} \mathrm{P}_{2}, \mathrm{P}_{2} \mathrm{P}_{3}$, \&c., the lines also being normal to the paper, we shall obtain a diffraction-grating which will give an image of $Q$ without aberration at $Q_{1}$.

We can thus determine the law according to which lines must be ruled on any cylindrical surface to give an aplanatic diffraction-image of a slit; for we require only to write down the equations to the ellipses and the surface and determine the points of intersection. We will solve the simple case when the curve $P_{1} P_{2}$ \&c. is a straight line parallel to $Q Q_{1}$. Take $Q Q_{1}$ as axis of $x$; let $a$ and $b$ be the semi-axes of one of the ellipses, suppose that which touches the line $\mathrm{P}_{1} \mathrm{P}_{2} \ldots$; then the semi major axes of the other ellipses are

$$
a+\frac{\lambda}{2}, a+\delta \ldots a+\frac{n \lambda}{2}, \text { \&c.; }
$$

while to find $b_{n}$, the semi minor axis of the $(n+1)$ th ellipse, we have

$$
\left(a+\frac{n \lambda}{2}\right)^{2}-l_{n}^{2}=a^{2}-l^{2} \cdot A l_{n}^{2}=l^{2}+n a \lambda+\frac{n^{2} \lambda^{2}}{4} .
$$

Let $Q Q_{1}=2 c$, then we have

$$
a^{2}=b^{2}+c^{2}, \text {. . . . . . }
$$

and the equation to the $(n+1)$ th ellipse is

$$
\frac{x^{2}}{a^{2}+n a \lambda+\frac{n^{2} \lambda^{2}}{4}}+\frac{y^{2}}{6^{2}+n a \lambda+\frac{n^{2} \lambda^{2}}{4}}=1 . .
$$

Let $x_{n}$ be the abscissa of the point in which this is cut by the line $y=b$, then

$$
x_{n}^{2}=\frac{\left(a^{2}+n a \lambda+\frac{n^{2} \lambda^{2}}{4}\right)\left(n a \lambda+\frac{n^{2} \lambda^{2}}{4}\right)}{l^{2}+n a \lambda+\frac{n^{2} \lambda^{2}}{4}} .
$$


Substituting for $a$ and giving $n$ the values $0,1,2,3, \& c$. in order, we can obtain values for $i_{0}, x_{1}, x_{2}$ \&c., and determine thus the position of the lines. A plane grating ruled in this manner would form at $Q_{1}$ without aberration an image of $Q$ for light of the given wave-length $\lambda$. Of course it would be open to the objection which holds against all such aplanatic arrangements, viz. that they are only good for light of one definite wave-length. If the grating were used for light of a different refrangibility, the image formed would suffer from aborration.

\section{A new Plotometer. $B y$ Sir John Coxroy, Bart., M.A.*}

Ilaving recently made a considerable number of photometric observations, and learnt by experience the difficulty which attends all such determinations, I venture to bring before the Soriety the description of a new form of photometer which aplears to possoss certain advantages over those in use. All such instruments, with the execption of the wedre-photometer, are essentially arrangements for comparing the illaminating-power of two lights, and therefore do not give alssolute measures; the one I propose describing is no exception to this general rule.

I lad intended to use, in some experiments on the amount of light reffected by metallic surfaces, the ordinary Bunsen's disk; but I found that, owing to the small size of the bosm of reffected light, it was impossible to make any satisfactory measurements with the disks in common use, and after trying various photometric arrangements I finally adopted a moditiaation of Ritchie's photoncter.

The various forms of shadow-photometers work well; but as the accuracy of the determination depends on the edge of the two shadows coinciding and yet not overlapping, it is necessary to have some arrangement for altering the distance between the screen and the shadow-producer, which adds to the complexity of the apparatus, except indeed when, as in Mr. Harcourt's photometer for gas-work, the variation in the relative intensities of the two lights is caused by the size of one of the flames being altered, and not, as in those arringe-

* Read April 28, 188:3. 